\title{
Effect of Boron Incorporation on Slow Interface Traps in $\mathrm{SiO}_{2} / 4 \mathrm{H}-\mathrm{SiC}$ Structures
}

\author{
Dai Okamoto - Mitsuru Sometani - Shinsuke Harada - Ryoji Kosugi • \\ Yoshiyuki Yonezawa · Hiroshi Yano
}

Received: date / Accepted: date

\begin{abstract}
The reason for the effective removal of interface traps in $\mathrm{SiO}_{2} / 4 \mathrm{H}-\mathrm{SiC}$ (0001) structures by boron (B) incorporation was investigated by employing lowtemperature electrical measurements. Low-temperature capacitance-voltage and thermal dielectric relaxation current measurements revealed that the density of electrons captured in slow interface traps in B-incorporated oxide is lower than that in dry and NO-annealed oxides. These results suggest that near-interface traps can be removed by $\mathrm{B}$ incorporation, which is considered to be an important reason for the increase in the field-effect mobility of 4H-SiC metal-oxide-semiconductor devices. A model for the passivation mechanism is proposed that takes account of stress relaxation during thermal oxidation.
\end{abstract}

Keywords $\mathrm{SiC}$. MOS interface - Near interface traps · Boron diffusion - Thermal dielectric relaxation current

\section{Introduction}

Silicon carbide (SiC) has attracted considerable attention over the last decade as a promising candidate for use in next-generation power devices for high-voltage and high-temperature applications. SiC power metaloxide--semiconductor field-effect transistors (MOSFETs)

D. Okamoto · H. Yano

Faculty of Pure and Applied Sciences, University of Tsukuba, 1-1-1 Tennodai, Tsukuba, 305-8573, Ibaraki, Japan

Tel.: +81-29-853-5600 (ex. 91881)

Fax: +81-29-853-5838

E-mail: okamoto.dai.gb@u.tsukuba.ac.jp

M. Sometani · S. Harada · R. Kosugi · Y. Yonezawa Advanced Power Electronics Research Center, National Institute of Advanced Industrial Science and Technology (AIST), Central 2, 1-1-1 Umezono, Tsukuba, 305-8568, Ibaraki, Japan have been commercialized and gradually put into practical use since the early 2010s. However, the channel resistance in the latest $4 \mathrm{H}-\mathrm{SiC}$ power MOSFETs still accounts for about half the total conduction loss. Despite many years of process development on $4 \mathrm{H}-\mathrm{SiC}$ MOS devices, the interface state density $\left(D_{\text {it }}\right)$ remains high, resulting in low field-effect mobility. In particular, 4H-SiC MOSFETs fabricated by dry oxidation on a (0001) Si-face exhibit a field-effect mobility below $10 \mathrm{~cm}^{2} \mathrm{~V}^{-1} \mathrm{~s}^{-1}[1]$, which hinders the development of planar-type $\mathrm{SiC}$ MOS devices. If we are to develop future-generation $\mathrm{SiC}$ power MOSFETs while achieving a low conduction loss, we must elucidate both the cause of the high interface state density and its passivation mechanisms.

Several annealing methods have been proposed for reducing $D_{\text {it }}$ and enhancing the field-effect mobility of $4 \mathrm{H}-\mathrm{SiC}$ MOSFETs. One of the best ways to reduce $D_{\text {it }}$ is the nitridation of the interface with $\mathrm{NO}$ or $\mathrm{N}_{2} \mathrm{O}$ gases [2-4]. Field-effective mobilities of $20-50 \mathrm{~cm}^{2} \mathrm{~V}^{-1} \mathrm{~s}^{-1}$ and good oxide reliability can be obtained by the nitridation of the (0001) Si-face of 4H-SiC [3,4] and this process currently constitutes the industrial standard. Further mobility enhancement up to around $170 \mathrm{~cm}^{2} \mathrm{~V}^{-1} \mathrm{~s}^{-1}$ has been achieved by sodium-enhanced oxidation on the (0001) Si-face of 4H-SiC [5]. In addition, we have reported that the incorporation of phosphorus $(\mathrm{P})$ into a $\mathrm{SiO}_{2} / 4 \mathrm{H}-\mathrm{SiC}$ interface results in a low $D_{\text {it }}$ and a high field-effect mobility of up to $89 \mathrm{~cm}^{2} \mathrm{~V}^{-1} \mathrm{~s}^{-1}$ on the (0001) Si-face of $4 \mathrm{H}-\mathrm{SiC}$ [6].

More recently, we have reported that the incorporation of boron (B) into a $\mathrm{SiO}_{2} / 4 \mathrm{H}-\mathrm{SiC}(0001)$ interface reduces $D_{\text {it }}$ close to the conduction band edge of $4 \mathrm{H}$ $\mathrm{SiC}$ [7]. A boron nitride (BN) planar diffusion source was used as the B source, and the fabricated MOSFETs exhibited a field-effect mobility of $102 \mathrm{~cm}^{2} \mathrm{~V}^{-1} \mathrm{~s}^{-1}$ [7]. 
In addition, Soler et al. reported the $4.5 \mathrm{kV} 4 \mathrm{H}-\mathrm{SiC}$ power MOSFETs with the B-doped gate oxide combined with a deposited $\mathrm{SiO}_{2}$, demonstrating both the low on-resistance and relatively stable threshold voltage [8]. Therefore, the $\mathrm{B}$ diffusion is one promising process for the next-generation $\mathrm{SiC}$ power devices. This technique is unique in terms of utilizing a group III element. The fact that $D_{\text {it }}$ can be reduced by B is in clear contrast to the many conventional passivation methods which are based on group $\mathrm{V}$ elements that act as donors in a $4 \mathrm{H}-\mathrm{SiC}$ substrate $[9,10]$. The mechanism behind the improved field-effect mobility by the B incorporation remains unclear, and detailed investigations are required.

Here, we would like to discuss possible origins of interface traps at the $\mathrm{SiO}_{2} / 4 \mathrm{H}-\mathrm{SiC}$ interface. Afanas'ev et al. suggested three possible origins for the interface states at the $\mathrm{SiO}_{2} / 4 \mathrm{H}-\mathrm{SiC}$ interface: (i) $\mathrm{Si}$ or $\mathrm{C}$ dangling bonds, (ii) $\mathrm{C}$ clusters, and (iii) near-interface traps (NITs) $[11,12]$. Of these, NITs may play a dominant role because they are considered to create trap levels close to the conduction band edge of $4 \mathrm{H}-\mathrm{SiC}$ at a high density [11-14]. It has been reported that NITs can be effectively removed by the incorporation of $\mathrm{N}$ [15], $\mathrm{Na}$ [16], K [17], and $\mathrm{P}$ [18] as revealed either by using low-temperature capacitance-voltage $(C-V)[15,18]$ or thermal dielectric relaxation current (TDRC) [16-18] measurements. It is interesting to note that improved field-effect mobilities can be obtained by utilizing oxides incorporating $\mathrm{N}[3,4], \mathrm{Na}[5]$, and $\mathrm{P}[6]$, and thus we can assume that the NIT density is closely related to the field-effect mobility. This makes it also important to investigate the nature of NITs in B-incorporated MOS structures for a comprehensive understanding of the origin of interface states and the passivation mechanisms in the $\mathrm{SiO}_{2} / 4 \mathrm{H}-\mathrm{SiC}$ interface structures.

In this study, we investigated the density of the trapped electrons in the NITs in the B-incorporated gate oxides in comparison with that in conventional dry and NO-annealed oxides. We employed $C-V$ measurements at a low temperature [14] and TDRC measurements [19] to analyze the density of NITs. We discuss a possible mechanism for the improved field-effect mobility based on the excess Si atoms near the interface and interface stress during oxidation, and propose a model of the passivation mechanism. Some researchers have pointed out that near-interface oxide traps are also located close to the $4 \mathrm{H}-\mathrm{SiC}$ valence band edge [20-22]. However, in this study, we focus on the NITs located close to the conduction band edge of $4 \mathrm{H}-\mathrm{SiC}$, which are strongly related to the channel mobility of $4 \mathrm{H}-\mathrm{SiC}$ MOSFETs. Thus, we evaluated n-type MOS capacitors to investigate the NITs close to the conduction band edge of $4 \mathrm{H}-\mathrm{SiC}$.

\section{Experimental details}

N-type MOS capacitors were fabricated on n-type $4^{\circ}$-off $4 \mathrm{H}-\mathrm{SiC}$ (0001) Si-face epitaxial layers with a net donor concentration of $1 \times 10^{16} \mathrm{~cm}^{-3}$. MOS capacitors with three different gate oxides were fabricated: (i) dry, (ii) NO-annealed, and (iii) B-incorporated oxides. After standard RCA cleaning, oxides approximately $47 \mathrm{~nm}$ thick were formed by dry oxidation at $1200{ }^{\circ} \mathrm{C}$ for $170 \mathrm{~min}$. For the dry + NO samples, post oxidation annealing was performed in 20\%-diluted NO (NO/Ar $=1.0 / 4.0$ slm) at around $1350{ }^{\circ} \mathrm{C}$ for $3 \mathrm{~min}$ using a cold-wall furnace [23]. For the dry + B-diffusion samples, the dry-oxidized substrate was put into a three-zone hotwall oxidation furnace and placed about $1 \mathrm{~mm}$ from a planar diffusion source (Saint-Gobain, BN-975) composed of $\mathrm{BN}$ and $3.5-6.5 \%$ boric oxide $\left(\mathrm{B}_{2} \mathrm{O}_{3}\right)$. The sample was then annealed at $950{ }^{\circ} \mathrm{C}$ for $10 \mathrm{~min}$ in an $\mathrm{O}_{2}(0.2 \mathrm{slm})$ and $\mathrm{Ar}(1.8 \mathrm{slm})$ gas mixture. The sample was subsequently annealed in $\mathrm{Ar}$ at $950{ }^{\circ} \mathrm{C}$ for $120 \mathrm{~min}$ without the $\mathrm{BN}$ plate. Al was evaporated to form gate and backside electrodes. The diameter of the gate electrodes was $400 \mu \mathrm{m}$. High-frequency $C-V$ measurements $(100 \mathrm{kHz}$ or $1 \mathrm{MHz})$ were implemented using an Agilent 4294A impedance analyzer. Low-frequency $(1 \mathrm{~Hz})$ $C-V$ measurements were performed using an Agilent ultra-low-frequency $C-V$ system, which consisted of an Agilent B1500A and an Agilent 33210A function generator. TDRC measurements were carried out at temperatures between 40 and $300 \mathrm{~K}$ with a constant heating rate of $0.333 \mathrm{~K} / \mathrm{s}(20 \mathrm{~K} / \mathrm{min})$ using a Keithley 4200 semiconductor characterization system equipped with preamps. The TDRC measurement is described in detail in Ref. [19].

\section{Results}

First, the $D_{\text {it }}$ distributions were estimated by employing the hi-lo $C-V$ method using $1 \mathrm{~Hz}$ and $1 \mathrm{MHz}$ curves. Figure 1 shows the $D_{\text {it }}$ distribution against the trap energy $(E)$ from the conduction band edge $\left(E_{\mathrm{c}}\right)$ of $4 \mathrm{H}-$ $\mathrm{SiC}$ for dry, NO-annealed, and B-incorporated oxides as basic information about the fabricated samples. Note that the estimated $D_{\text {it }}$ values only reflect the interface traps that can respond to the $1 \mathrm{~Hz}$ to $1 \mathrm{MHz}$ frequency range due to the limitation of the Hi-Lo method [24, $25]$, and faster and shallower interface traps were not fully evaluated. Nevertheless, it is clear that B incorporation can reduce $D_{\text {it }}$ by more than an order of magnitude. The peak field-effect mobility values for the $4 \mathrm{H}-$ SiC MOSFETs fabricated on the (0001) Si-face were 4, 17 , and $102 \mathrm{~cm}^{2} \mathrm{~V}^{-1} \mathrm{~s}^{-1}$ for dry, NO-annealed, and Bincorporated oxides, respectively (not discussed in this 


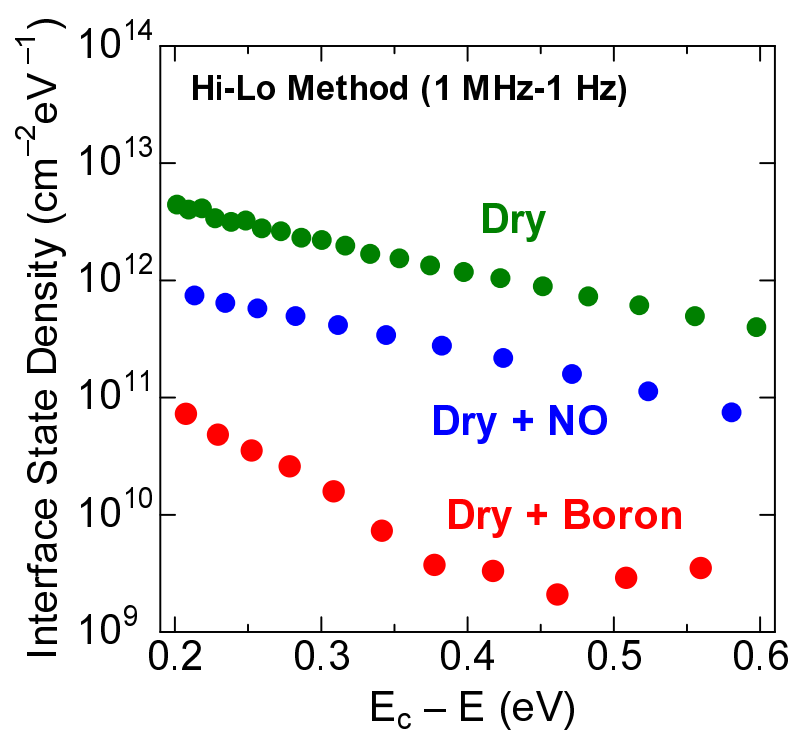

Fig. 1 Distributions of interface state density close to the conduction band edge of $4 \mathrm{H}-\mathrm{SiC}$ for dry, NO-annealed, and B-incorporated oxides.

paper). The characteristics of B-diffused MOSFETs are described in Ref. [7].

One of the most convenient ways to investigate the slow interface traps at the $\mathrm{SiO}_{2} / 4 \mathrm{H}-\mathrm{SiC}$ interface is to measure the start-voltage dependence of $C-V$ curves at a sufficiently low temperature, as originally proposed by Afanas'ev et al. [14]. Figure 2 shows the start-voltage dependence of high-frequency $(100 \mathrm{kHz}) C-V$ curves measured at $80 \mathrm{~K}$ for the (a) dry, (b) NO-annealed, and (c) B-incorporated oxides. The gate voltage was swept from accumulation to depletion, and the $C-V$ measurements were iterated while the start voltage was increased from 2 to $26 \mathrm{~V}$ in $2-\mathrm{V}$ steps. The sweep rate was approximately $0.5 \mathrm{~V} / \mathrm{s}$. We used $100 \mathrm{kHz}$ as the measurement frequency to avoid the decrease in accumulation capacitance due to series resistance. Parallel shifts in the $C-V$ curves in the positive direction were observed for the dry and NO-annealed oxides that depended on the start voltage, suggesting that the electrons were captured in the traps located on the oxide side of the interface $[14,15]$. The electrons are thought to be captured by the NITs whose energy position is aligned close to the conduction band edge of $4 \mathrm{H}-\mathrm{SiC}$ [11-14] because the Fermi level is very close to the conduction band edge in strong accumulation conditions. The time constant of the NITs is long because of the separation from the interface [11-13]. The electrons trapped in the NITs act like negative fixed charges in the oxide at $80 \mathrm{~K}$, and, as a result, a parallel shift of the $C-V$ curves in the positive direction was observed. The

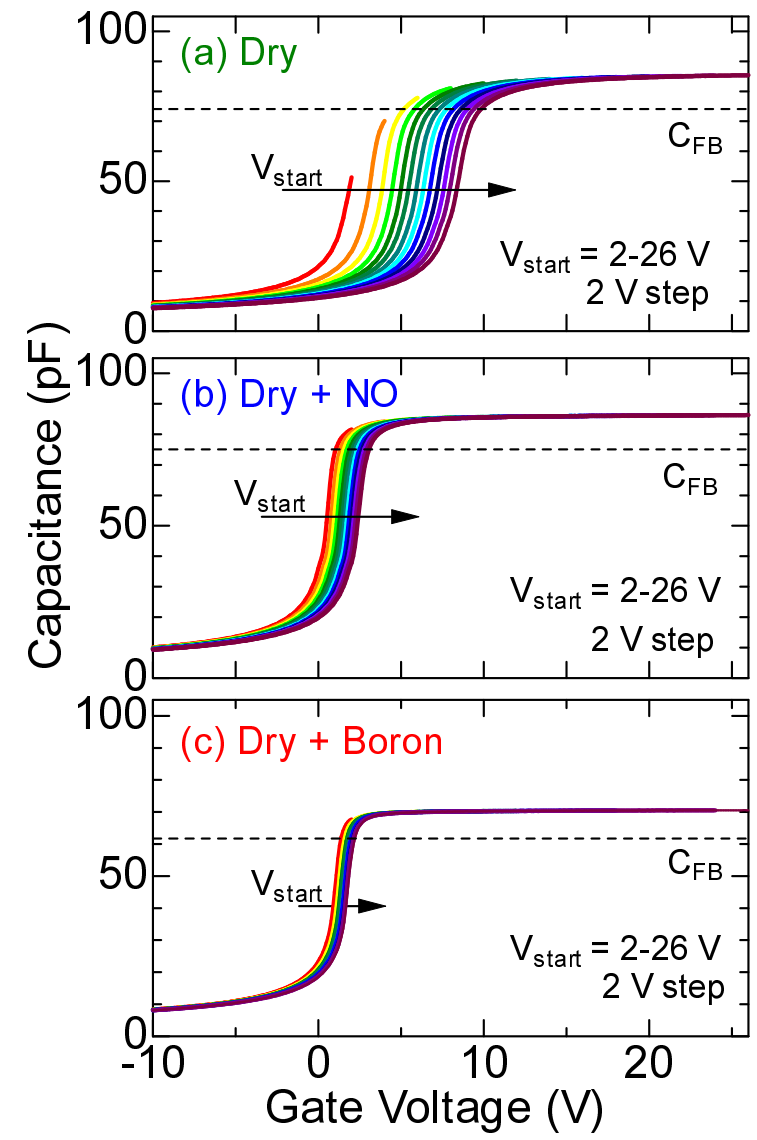

Fig. 2 Start-voltage dependence of high-frequency $(100 \mathrm{kHz})$ $C-V$ curves measured at $80 \mathrm{~K}$ for (a) dry, (b) NO-annealed, and (c) B-incorporated oxides. The gate voltage was swept from accumulation to depletion. The start voltage was increased from 2 to $26 \mathrm{~V}$ in $2-\mathrm{V}$ steps. The dashed lines denote flat-band capacitance $\left(C_{\mathrm{FB}}\right)$ at $80 \mathrm{~K}$.

start voltage dependence effectively decreased for the Bincorporated oxide. This result indicates that the density of the NITs decreased more significantly in the Bincorporated samples than in the NO-annealed samples. The slight shift in the low-temperature $C-V$ curves in the B-incorporated sample may be due to the imperfect passivation of the slow traps. Even though there is a slight shift in the $C-V$ curves for the B sample, the shift is always smaller than for the NO samples at $80 \mathrm{~K}$.

TDRC measurements were carried out to estimate the slow interface trap density. The heating rate $(\beta)$ was $0.333 \mathrm{~K} / \mathrm{s}$ and the discharging voltage $\left(V_{\text {dis }}\right)$ was $-5 \mathrm{~V}$. The discharging voltage is not so important because we have confirmed that almost the same TDRC curves can be obtained at $V_{\text {dis }}=-10 \mathrm{~V}$. Figure 3 shows the TDRC curves at which the oxide electric field $E_{\mathrm{ox}}=3.0 \pm 0.1$ $\mathrm{MV} / \mathrm{cm}$. It is generally difficult to determine the oxide electric field precisely, but the charges in the oxide 


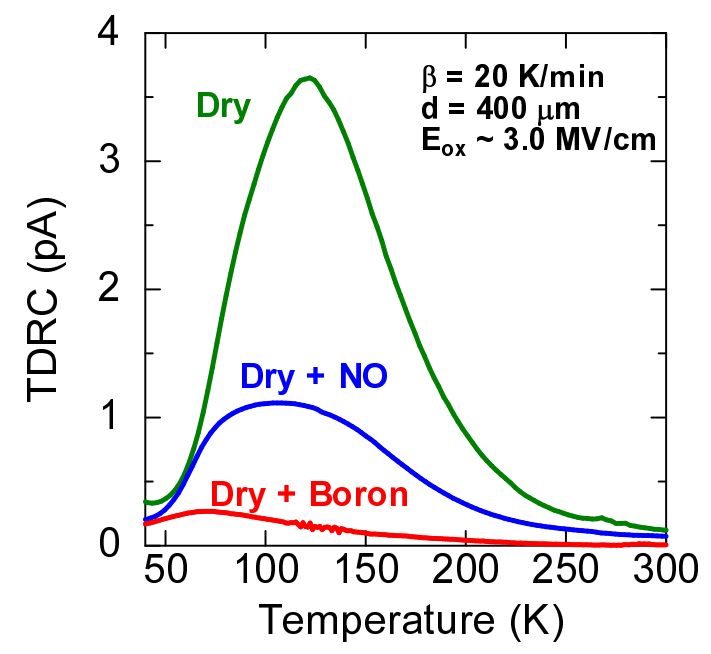

Fig. 3 TDRC spectra for dry, NO-annealed, and Bincorporated oxides. The oxide electric field during charging was approximately $3.0 \pm 0.1 \mathrm{MV} / \mathrm{cm}$. The heating rate $(\beta)$ was $0.333 \mathrm{~K} / \mathrm{s}$. The discharging voltage $\left(V_{\mathrm{dis}}\right)$ was $-5 \mathrm{~V}$.

seem to affect the $E_{\text {ox }}$ value. Thus, in this study, we assumed that $E_{\mathrm{ox}} \approx\left(V_{\mathrm{G}}-V_{\mathrm{FB}, \mathrm{RT}}\right) / t_{\mathrm{ox}}$, where $V_{\mathrm{G}}$ is the gate voltage and $V_{\mathrm{FB}, \mathrm{RT}}$ is the flat-band voltage measured at $1 \mathrm{MHz}$ at room temperature, and $t_{\mathrm{ox}}$ is the oxide thickness determined by the accumulation capacitance assuming the dielectric constant to be 3.9. A large TDRC spectrum can be found in the dry sample due to the negative charges emitted from the slow interface traps. Rudenko et al. suggested that the TDRC spectrum observed in the 50-250 K temperature range is a signal caused by the electrons emitted from the NITs spatially located in the $\mathrm{SiO}_{2}$ near the $\mathrm{SiO}_{2} / 4 \mathrm{H}-$ $\mathrm{SiC}$ interface, rather than fast interface states that reside exactly at the interface [19]. The parallel shift observed in the $C-V$ measurements at a low temperature also supports the detection of NITs [19]. The TDRC spectrum was reduced by $\mathrm{NO}$ annealing, but its effect was insufficient to passivate the active NITs. On the other hand, a much smaller TDRC spectrum was observed for the B-incorporated oxide, indicating that the NITs were effectively passivated. The TDRC curve for the B-incorporated sample is similar to those of the Na-incorporated [16] and P-incorporated samples [18], implying that their passivation mechanisms are similar.

By integrating a TDRC curve, we can estimate the density of the thermally stimulated electrons from the NITs [19]. The emitted charge density $N_{\mathrm{em}}$ is described as

$N_{\mathrm{em}}=\frac{1}{\beta} \int_{T_{\text {low }}}^{T_{\text {high }}}\left|J_{\operatorname{TDRC}}(T)\right| d T$



Fig. 4 Density of emitted electrons from NITs as a function of the estimated electric field across the oxide during charging. The dashed line denotes the total accumulated charge at the $\mathrm{SiO}_{2} / 4 \mathrm{H}-\mathrm{SiC}$ interface during charging.

where, $T_{\text {high }}$ is the upper limit of the evaluable temperature, $T_{\text {low }}$ is the lower limit of the evaluable temperature, $J_{\text {TDRC }}$ is the thermally-stimulated current density $\left(\mathrm{A} / \mathrm{cm}^{2}\right)$, and $\beta$ is the ramp rate during heating $(\mathrm{K} / \mathrm{sec})[19]$. The TDRC spectra were integrated in the 40 to $300 \mathrm{~K}$ temperature range because the constant heating rate is realized only at temperatures above $40 \mathrm{~K}$ in our measurement setup.

Figure 4 shows the $E_{\text {ox }}$ dependence of the thermally stimulated charge density calculated with Eq. (1). As $E_{\text {ox }}$ increases, the emitted charge density increases, particularly for the dry oxide. As the charging voltage increases, the electron density in the accumulation layer of an n-type MOS capacitor increases, and then the vast majority of the accumulated electrons are captured by the NITs for the dry oxide. The electron density in the accumulation layer was roughly estimated with $\epsilon_{\mathrm{ox}} E_{\mathrm{ox}} / q$ and is indicated in Fig. 4 with a broken line, where $\epsilon_{\mathrm{ox}}$ is the permittivity of the oxide and $q$ is the elementary charge. Most of the accumulated electrons were captured by the slow traps for the dry oxide, whereas it is clear that the trapped charge density was reduced substantially by the $\mathrm{B}$ incorporation. For dry oxide, the thermally stimulated electron density does not reach the saturation value in the measured bias range, and the NIT density is at least $6 \times 10^{12} \mathrm{~cm}^{-2}$ and may exceed $1 \times 10^{13} \mathrm{~cm}^{-2}$. The NIT density of the NO-annealed oxide decreased by more than a factor of two. The NIT density was further reduced by the B-incorporated oxide. The reduction in the TDRC spectra is in good agreement with the early results ob- 
tained with $\mathrm{Na}$ and $\mathrm{P}$ incorporation [16,18], suggesting that the NIT density is strongly related to the field effect mobility.

\section{Discussion}

The two experiments described above revealed that the acceptor-type slow electron traps reside close to the conduction band edge of $4 \mathrm{H}-\mathrm{SiC}$ particularly for the dry oxide, which agrees with the findings of early studies [14-19]. These slow traps can be effectively passivated by $\mathrm{B}$ incorporation. The observed slow interface traps cannot be explained solely by the fast interface states that reside exactly at the interface, and it is necessary to take the existence of NITs into account $[14,19]$. It has also been shown that the electron capture cross-section of the interface traps varies by several orders of magnitude and the value is small near the conduction band edge $[26,27]$. This implies that the traps are located in the oxide near the interface, and the capture cross-section is small because of the distance from the interface. If the electrons are captured by the NITs, the channel electron density is reduced and the estimated field-effect mobility decreases. Although the NITs are believed to be located in the oxide within a few nanometers of the interface $[11,13]$, some of them may also impede the channel electron transport due to remote Coulomb scattering.

Afanas'ev et al. suggested that the NITs are inherent defects in $\mathrm{SiO}_{2}$ located near the interface, and their energy position is $2.8 \mathrm{eV}$ below the conduction band edge of $\mathrm{SiO}_{2}[11,13]$. Schöner et al. also suggested that the defect levels close to the conduction band edge of $\mathrm{SiC}$ are identical for $4 \mathrm{H}-, 6 \mathrm{H}-$, and $15 \mathrm{R}-\mathrm{SiC}$, and they are energetically pinned at around $2.9 \mathrm{eV}$ above the valence band edge of all the $\mathrm{SiC}$ polytypes [28]. The microscopic nature of NITs is still unknown, but excess $\mathrm{Si}$ atoms in $\mathrm{SiO}_{2}$ [11-14], $\mathrm{C}=\mathrm{C}$ dimers [29], and $\mathrm{Si}_{2}-\mathrm{C}-\mathrm{O}$ defects [30] have been proposed as possible candidates. However, the experimentally determined fact that the energy position of NITs is identical for both $\mathrm{Si}$ and $\mathrm{SiC}$ substrates $[11,13]$ cannot be explained by the C-related defect structures. Thus, it may be reasonable to assume that the origin of NITs is Si-related defects as suggested by Afanas'ev et al. [11-14].

Although there is still a lack of convincing evidence for the physical origin of the NITs, their creation may be related to interface stress $[15,18]$. We consider that one possible origin for the NITs is excess Si atoms accumulated near the interface during thermal oxidation caused by interface stress. Si atoms are believed to be released from the interface during thermal oxidation both for the $\mathrm{Si}$ and $\mathrm{SiC}$ substrates to release the interface stress caused by volume expansion [3133]. During the thermal annealing used for incorporating $\mathrm{B}$ atoms, the oxide thickness increased from 47 to $65 \mathrm{~nm}$ [7], meaning that there was additional thermal oxidation of the $4 \mathrm{H}-\mathrm{SiC}$ substrate. A new interface is formed during this additional thermal oxidation, and $\mathrm{B}$ atoms are incorporated into the interface. $\mathrm{B}_{2} \mathrm{O}_{3}$ is known as a network former that reduces the connectivity of $\mathrm{SiO}_{2}$ network [34], which is similar to the case of $\mathrm{P}_{2} \mathrm{O}_{5}$ incorporation [35]. Thus, the interface stress that occurs during thermal oxidation as a result of the volume expansion may be easily released because of the flexible oxide network realized by B incorporation [34]. This may lead to reduced NIT density and consequently high field-effect mobility in 4H-SiC MOSFETs. This interpretation is very similar to the case of $\mathrm{P}$ incorporation [18]. However, this result is very important because both the elements have similar effect for reducing the slow traps. $\mathrm{P}$ is in group $\mathrm{V}$ in the periodic table, whereas B is in group III. Therefore, the passivation of the slow interface traps is not related to the group number in the periodic table, but it may be related to the interface stress. Although the model discussed in this paper is currently speculative, this result will facilitate future investigations from a new perspective designed to improve the $\mathrm{SiO}_{2} / 4 \mathrm{H}-\mathrm{SiC}$ interface quality. In light of the interface stress, further experimental and theoretical investigations are needed to verify the proposed model and identify the passivation mechanisms that account for the high field-effect mobility obtained by $\mathrm{B}$ incorporation.

\section{Conclusions}

In this study, low-temperature $C-V$ and TDRC measurements were employed to investigate the change in NIT density caused by B incorporation. The most important result we obtained is that the NITs were significantly reduced by $\mathrm{B}$ incorporation, which is similar to the case of $\mathrm{P}$ and $\mathrm{Na}$ incorporation by which high fieldeffect mobilities can be obtained. We discussed a model in which the possible origin of the NITs is accumulated Si atoms resulting from interface stress caused by volume expansion during thermal oxidation. We consider that $\mathrm{B}$ incorporation reduces the interface stress during thermal oxidation, thus lowering the NIT density. Therefore, we suggest that it is important to find a technology that minimizes interface stress during thermal oxidation to improve the field-effect mobility of $4 \mathrm{H}-\mathrm{SiC}$ MOSFETs.

Acknowledgements This work was supported by JSPS KAKENHI Grant Number 26820136 [Grant-in-Aid for Young Sci- 
entists (B)]. We thank T. Fuyuki and A. Tani at Nara Institute of Science and Technology (NAIST) for their help with the low-temperature measurements. We also thank the AIST $\mathrm{SiC}$ Power Device Team for useful discussions.

\section{References}

1. S. Harada, R. Kosugi, J. Senzaki, W. J. Cho, K. Fukuda, K. Arai, S. Suzuki, J. Appl. Phys. 91, 1568 (2002)

2. H. Li, S. Dimitrijev, H. B. Harrison, D. Sweatman, Appl. Phys. Lett. 70, 2028 (1997)

3. G. Y. Chung, C. C. Tin, J. R. Williams, K. McDonald, R. K. Chanana, R. A. Weller, S. T. Pantelides, L. C. Feldman, O. W. Holland, M. K. Das, J. W. Palmour, IEEE Electron Device Lett. 22, 176 (2001)

4. J. Rozen, A. C. Ahyi, X. Zhu, J. R. Williams, L. C. Feldman, IEEE Trans. Electron Devices 58, 3808 (2011)

5. G. Gudjónsson, H. Ö. Ólafsson, F. Allerstam, P.-Å. Nilsson, E. Ö. Sveinbjörnsson, H. Zirath, T. Rödle, R. Jos, IEEE Electron Device Lett. 26, 96 (2005)

6. D. Okamoto, H. Yano, K. Hirata, T. Hatayama, T. Fuyuki, IEEE Electron Device Lett. 31, 710 (2010)

7. D. Okamoto, M. Sometani, S. Harada, R. Kosugi, Y. Yonezawa, H. Yano, IEEE Electron Device Lett. 35, 1176 (2014)

8. V. Soler, M. Cabello, J. Montserrat, J. Rebollo, J. Millán, P. Godignon, M. Berthou, E. Bianda, A. Mihaila, 4.5kV SiC MOSFET with Boron Doped Gate Dielectric, Proc. 28th Int. Symp. Power Semiconductor Devices and ICs (ISPSD), Prague, Czech Republic, pp. 283-286, June 2016.

9. P. Fiorenza, F. Giannazzo, M. Vivona, A. La Magna, F. Roccaforte, Appl. Phys. Lett. 103, 153508 (2013)

10. G. Liu, A. C. Ahyi, Y. Xu, T. Isaacs-Smith, Y. K. Sharma, J. R. Williams, L. C. Feldman, S. Dhar, IEEE Electron Device Lett. 34, 181 (2013)

11. V. V. Afanas'ev, M. Bassler, G. Pensl, M. Schulz, Phys. Status Solidi A 162, 321 (1997)

12. G. Pensl, M. Bassler, F. Ciobanu, V. V. Afanas'ev, H. Yano, T. Kimoto, H. Matsunami, Mater. Res. Soc. Symp. Proc. 640, H3.2.1 (2001)

13. V. V. Afanas'ev, A. Stesmans, Phys. Rev. Lett. 78, 2437 (1997)

14. V. V. Afanas'ev, A. Stesmans, M. Bassler, G. Pensl, M. J. Schulz, Appl. Phys. Lett. 76, 336 (2000)

15. V. V. Afanas'ev, A. Stesmans, F. Ciobanu, G. Pensl, K. Y. Cheong, S. Dimitrijev, Appl. Phys. Lett. 82, 568 (2003) 16. F. Allerstam, H. Ö. Ólafsson, G. Gudjónsson, D. Dochev, E. Ö. Sveinbjörnsson, T. Rödle, R. Jos, J. Appl. Phys. 101, 124502 (2007)

17. P. G. Hermannsson, E. Ö. Sveinbjörnsson, Mater. Sci. Forum 717-720, 761 (2012)

18. D. Okamoto, H. Yano, T. Hatayama, T. Fuyuki, Appl. Phys. Lett. 96, 203508 (2010)

19. T. E. Rudenko, I. N. Osiyuk, I. P. Tyagulski, H. Ö. Ólafsson, E. Ö. Sveinbjörnsson, Solid-State Electron. 49, $545(2005)$

20. A. J. Lelis, R. Green, D. B. Habersat, M. El, IEEE Trans. Electron Devices 62, 316 (2015)

21. C. T. Yen, C. C. Hung, H. T. Hung, C. Y. Lee, L. S. Lee, Y. F. Huang, F. J. Hsu, Appl. Phys. Lett. 108, 012106 (2016)

22. P. Fiorenza, A. L. Magna, M. Vivona, F. Roccaforte, Appl. Phys. Lett. 109, 012102 (2016)

23. R. Kosugi, K. Fukuda, K. Arai, Mater. Sci. Forum 483485, $669(2005)$
24. H. Yoshioka, T. Nakamura, T. Kimoto, J. Appl. Phys. 111, $014502(2012)$

25. A. V. Penumatcha, S. Swandono, J. A. Cooper, IEEE Trans. Electron Devices 60, 923 (2013)

26. X. D. Chen, S. Dhar, T. Isaacs-Smith, J. R. Williams, L. C. Feldman, P. M. Mooney, J. Appl. Phys. 103, 033701 (2008)

27. N. Yoshida, E. Waki, M. Arai, K. Yamasaki, J. H. Han, M. Takenaka, S. Takagi, Thin Solid Films 557, 237 (2014)

28. R. Schörner, P. Friedrichs, D. Peters, D. Stephani, IEEE Electron Device Lett. 20, 241 (1999)

29. J. M. Knaup, P. Deák, Th. Frauenheim, A. Gali, Z. Hajnal, W. J. Choyke, Phys. Rev. B 72, 115323 (2005)

30. F. Devynck, A. Alkauskas, P. Broqvist, A. Pasquarello, Phys. Rev. B 84, 235320 (2011)

31. H. Kageshima, K. Shiraishi, Phys. Rev. Lett. 81, 5936 (1998)

32. Y. Hijikata, H. Yaguchi, S. Yoshida, Appl. Phys. Express 2, 021203 (2009)

33. Y. Hijikata, H. Yaguchi, S. Yoshida, Mater. Sci. Forum 615-617, 489 (2009)

34. S. Miyazaki, K. Morino, M. Hirose, Solid-State Phenom. 76-77, 149 (2001)

35. K. Morino, S. Miyazaki, M. Hirose, Phosphorus incorporation in ultrathin gate oxides and its impact to the network structure, Ext. Abst. of 1997 Int. Conf. Solid State Device and Materials (SSDM), Hamamatsu, Japan, pp. 18-19, Sep. 1997. 